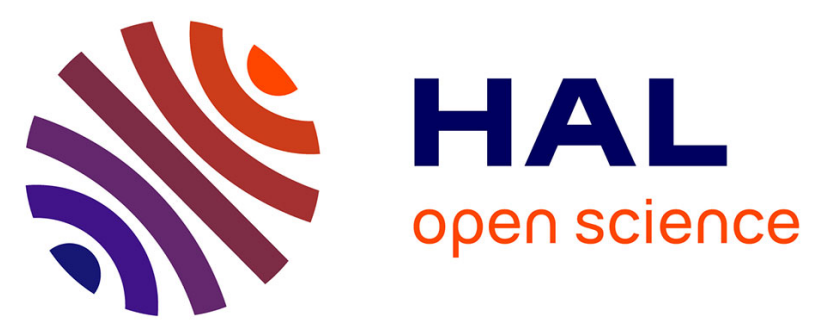

\title{
A temperature-sensitive tracer suitable for two-colour laser-induced fluorescence thermometry applied to evaporating fuel droplets
}

\author{
Valérie Deprédurand, Petre Miron, Alexandre Labergue, Michel Wolff, \\ Guillaume Castanet, Fabrice Lemoine
}

\section{To cite this version:}

Valérie Deprédurand, Petre Miron, Alexandre Labergue, Michel Wolff, Guillaume Castanet, et al.. A temperature-sensitive tracer suitable for two-colour laser-induced fluorescence thermometry applied to evaporating fuel droplets. Measurement Science and Technology, 2008, 19, pp.105403 - 105403. 10.1088/0957-0233/19/10/105403 . hal-01570410

\section{HAL Id: hal-01570410 \\ https://hal.univ-lorraine.fr/hal-01570410}

Submitted on 29 Jul 2017

HAL is a multi-disciplinary open access archive for the deposit and dissemination of scientific research documents, whether they are published or not. The documents may come from teaching and research institutions in France or abroad, or from public or private research centers.
L'archive ouverte pluridisciplinaire HAL, est destinée au dépôt et à la diffusion de documents scientifiques de niveau recherche, publiés ou non, émanant des établissements d'enseignement et de recherche français ou étrangers, des laboratoires publics ou privés. 


\title{
A temperature-sensitive tracer suitable for two-colour laser-induced fluorescence thermometry applied to evaporating fuel droplets
}

\author{
V Deprédurand, P Miron, A Labergue, M Wolff, G Castanet \\ and $F$ Lemoine
}

LEMTA, Nancy-Université, CNRS, 2, Avenue de la forêt de Haye, BP 160,

F-54504 Vandoeuvre-lès-Nancy, France

\begin{abstract}
This paper reports an extensive spectroscopic study of the fluorescence of pyrromethene 597-8C9, which can be used as a dye in two-colour laser-induced fluorescence thermometry. This technique requires a single dye and two spectral bands of detection to measure the droplet temperature. The selection of a fluorescent tracer is a critical issue. To achieve measurements with a reasonable accuracy, a tracer should exhibit a fluorescence spectrum that is particularly dependent on the temperature. Another important feature is the possibility of using the same dye and the same optical components with a large variety of fuels, including multi-component fuels which are commonly used in industrial applications, which was not possible with usual tracers like rhodamine B. This paper demonstrates that pyrromethene 597-8C9 is relevant for measuring the temperature of droplets made of alkanes (dodecane), ketones (3-pentanone) or alcohols (ethanol). It can also handle mixtures of ethanol and 3-pentanone. In this study, demonstration experiments are performed on droplets injected into a hot air chamber $(645 \mathrm{~K})$.
\end{abstract}

Keywords: laser-induced fluorescence, droplet, evaporation, pyrromethene

\section{Introduction}

Numerous studies have considered laser-induced fluorescence (LIF) as a powerful non-intrusive temperature measurement technique in fluid flows. Coolen et al [1] developed a planar laser-induced fluorescence (PLIF) technique, using rhodamine $\mathrm{B}$ as a fluorescent tracer, to investigate the development of natural convection flows. Lemoine et al [2] studied heat transfers in turbulent jets by measuring cross-correlations between velocity and temperature fluctuations. Measurements were performed by combining laser Doppler velocimetry and LIF, rhodamine B being also the fluorescent tracer. In these examples, one single fluorescent dye and one spectral band of detection are used, which presents some constraints. To ensure dependence of the fluorescence signal on solely the temperature, the dye concentration must remain constant and fluorescence has to be collected from a volume that is kept constant during the experiment. Furthermore, the incident laser intensity must also be kept constant for the same reason. However, when moving droplets are considered, these conditions are never fulfilled. The presence of an air/liquid interface modifies the distribution of the laser energy within the droplets in such a way that it is a function of the position of the droplet in the laser excitation volume.

Copetta and Rogers [3] provided a review of temperaturesensitive tracers and showed the interest in using ratiometric techniques. The temperature is determined from the ratio of the signal of two dyes, which have highly different temperature sensitivities. The emission spectra of the two dyes should differ enough to make possible the separation of the fluorescence light emitted by each tracer. Sakakibara and Adrian [4] pioneered the development of techniques based on two-colour and two-dye laser-induced fluorescence. In principle, one of the dyes should be sensitive to temperature (e.g. rhodamine B) whereas the other should be independent 
Table 1. Comparison between rhodamine B, kiton red and pyrromethene 597-8C9 on the basis of some practical issues related to their introduction in 2cLIF thermometry.

\begin{tabular}{lll}
\hline Remarkable properties & Pyrromethene 597-8C9 & Kiton red or rhodamine B \\
\hline Solubility in water & No & Yes \\
Solubility in alkanes & Yes & No \\
Solubility in ketones or alcohols & Yes & Yes \\
Shape of the fluorescence spectrum sensitive to $\mathrm{pH}$ & Not applicable & Yes \\
Shape of the fluorescence spectrum sensitive to temperature & Yes & Yes \\
Shape of the fluorescence spectrum sensitive to composition & No in certain cases & Yes \\
Temperature sensitivity depends on composition & No in certain cases & Yes \\
Suitable level of signal on the two spectral bands of detection & Yes & No \\
\hline
\end{tabular}

of or weakly sensitive to this parameter (e.g. rhodamine 110). Applications of this technique can be also found in [5], who studied Rayleigh-Benard convective flows. The advantage of this method is that the laser intensity and probe volume size dependences are eliminated. However, in addition to the temperature, the ratio between the signals emitted by the two dyes is also a function of the dye concentration ratio which must not vary during the measurement.

The two-colour laser-induced fluorescence technique uses a single fluorescent tracer and two spectral bands of detection. It has therefore the same advantages as the two-dye laserinduced fluorescence method without the problem of the variation of the dye concentration. The technique is perfectly suitable for measuring the temperature of droplets. It was developed first by Lavieille et al [6] to address the case of monodisperse droplets of ethanol streaming linearly.

Rhodamine B or kiton red (also called sulforhodamine B) are widely used [2,3] to measure temperature because of their high sensitivity to temperature and the fact that their fluorescence can be easily induced by commercial lasers, such as the argon ion laser $(\lambda=514.5 \mathrm{~nm})$ and the doubled Nd-Yag laser $(\lambda=532 \mathrm{~nm})$. Furthermore, they are highly soluble in water and in a wide range of solvents such as acetone, ethanol and methanol, but notably not in alkanes.

To overcome this limitation in alkanes, Wolff et al [10] used pyrromethene 597-8C9. The latter was dissolved in ISO 4113 oil (Shell normafluid that mimics diesel) to characterize the temperature of droplets in a spray injected under high pressure.

Since then, pyrromethene 597-8C9 has emerged as a promising fluorescent tracer to study fuel droplet evaporation where there is a need to investigate a wide range of fuels such as alkanes, ketones, aromatics, alcohols or their mixtures. Some criteria to select a suitable fluorescent dye for two-colour thermometry are summarized in table 1.

One drawback of rhodamine B or kiton red is that the overall shape of the fluorescence spectrum is affected by $\mathrm{pH}$ changes $[7,8]$ and more generally by composition variations of the liquid of interest [9]. In the case of pyrromethene 597$8 \mathrm{C} 9$, the fluorescence spectrum and the temperature sensitivity of its fluorescence response are almost independent of the mixture composition in many cases. In addition, in the case of rhodamine B or kiton red, the signal level collected on the first spectral band of detection, located in the green-yellow part of the spectrum [6], is generally relatively low. For pyrromethene
597-8C9, this signal level is more balanced between the two spectral bands.

The spectroscopic properties of pyrromethene 597-8C9 dissolved in pure liquids (3-pentanone, ethanol, dodecane) or in mixtures (ethanol/3-pentanone) are detailed. Finally, the reliability of the technique is tested by carrying out some experiments on monodispersed droplets made of different fuels (dodecane, 3-pentanone, ethanol and mixtures of ethanol and 3 -pentanone) injected in a hot air chamber (645 K).

\section{Two-colour laser-induced fluorescence: physical principles and governing equations}

Only the outlines of the principles of the two-colour laserinduced fluorescence are presented in this section. A comprehensive survey of the technique can be found in previous publications of Lavieille et al $[6,11]$. The liquid is preliminarily seeded with a low concentration (a few $\mathrm{mg}^{-1}$ ) of a fluorescent tracer which is temperature sensitive. The fluorescent tracer is generally an organic dye which can be efficiently dissolved in the liquid. The case of pyrromethene 597-8C9 will be investigated in the present paper. A key feature of the present application is that the temperature sensitivity of the fluorescence emission is wavelength dependent for some organic dyes [8]. The fluorescence intensity $I_{f}\left(\mathrm{~W} \mathrm{~m} \mathrm{~m}^{-2}\right)$ at the wavelength $\lambda$ collected by a detector is expressed as $[2,10]$

$$
I_{f}(\lambda)=K_{\text {opt }}(\lambda) K_{\text {spec }}(\lambda) V_{c} I_{0} C \mathrm{e}^{\beta(\lambda) / T}
$$

where $K_{\text {opt }}$ is an optical constant (dimensionless), $K_{\text {spec }}\left(\mathrm{mol}^{-1}\right)$ is a constant depending solely on the spectroscopic properties of the fluorescent tracer in its environment (i.e. the fuel), $I_{0}$ is the laser excitation intensity $\left(\mathrm{W} \mathrm{m}^{-2}\right), C$ is the molecular tracer concentration $\left(\mathrm{mol} \mathrm{L}^{-1}\right), T$ is the absolute temperature $(\mathrm{K})$ and $V_{c}$ is the fluorescence probe volume $\left(\mathrm{m}^{3}\right) . V_{c}$ is defined as the intersection between the illuminated part of the droplet volume and the field of view of the detection optics. The factor $\beta(\lambda)$ characterizes the temperature dependence of the fluorescence intensity at the wavelength $\lambda$. The exponential accounts for the temperature dependence of the absorption cross-section (related to the Boltzmann distribution of the energy levels) and the quenching rate, following an Arrhenius law.

The signal is detected on a spectral band $\left(\lambda_{i 1} ; \lambda_{i 2}\right), i$ being the spectral band index. The resulting fluorescence intensity 
on the $i$ th spectral band can be approximated by a second-order polynomial $[10,11]$ :

$$
\begin{aligned}
I_{f i} & =\int_{\lambda_{i 1}}^{\lambda i 2} K_{\mathrm{opt}}(\lambda) K_{\mathrm{spec}}(\lambda) V_{c} I_{0} C \mathrm{e}^{\beta(\lambda) / T} \mathrm{~d} \lambda \\
& \approx K_{\mathrm{opt} i} K_{\mathrm{spec} i} V_{c} I_{0} C \mathrm{e}^{\frac{a_{i}}{T}+\frac{b_{i}}{T^{2}}}
\end{aligned}
$$

where $K_{\mathrm{opt} i}$ and $K_{\mathrm{spec} i}$ are the optical and spectroscopic constants related to the spectral band $i$. The integral term in equation (2) is necessary because $\beta$ varies significantly over the spectral bandwidth, which is typically on the order of $20 \mathrm{~nm}$. The second-order approximation $\mathrm{e}^{\frac{a_{i}}{T^{2}}+\frac{b_{i}}{T}}$, which is purely empirical, is generally sufficient to account for the variation of the fluorescence signal integrated on a spectral band as a function of temperature. The parameters $a_{i}$ and $b_{i}$ are temperature sensitivity coefficients for the spectral band $i$. In order to measure the temperature of moving and potentially evaporating droplets, the parameters $C V_{c}$ and $I_{0}$ must be removed. The measurement volume is constantly changing when the droplet crosses the laser beam. This volume corresponds indeed to the triple intersection between the volume of excitation by the laser beam, the volume of detection by the receiving optics and the droplet. The intensity distribution of the laser inside the droplet depends on the droplet shape and on its position relative to the beam due to the lensing of the droplet interface. In the case of an evaporating droplet, the concentration of the fluorescent tracer is likely to vary. To overcome these problems, the fluorescence intensity is detected on two spectral bands characterized by temperature sensitivities as different as possible. The fluorescence ratio between both fluorescence intensities collected on the two spectral bands is given by

$$
R_{f}=\frac{I_{f 1}}{I_{f 2}}=\frac{K_{\mathrm{opt} 1}}{K_{\mathrm{opt} 2}} \frac{K_{\text {spec } 1}}{K_{\text {spec } 2}} \mathrm{e}^{\frac{a_{1}-a_{2}}{T}+\frac{b_{1}-b_{2}}{T^{2}}} .
$$

This ratio is totally independent of the dimensions of the probe volume. The influence of the local laser excitation intensity and tracer concentration is also totally eliminated. Finally, a reference ratio $R_{\text {ref }}$ is taken once in a place where the temperature can be safely known. The temperature $T_{\text {ref }}$ is found using a thermocouple, which allows replacement of the constants in equation (3):

$$
\begin{aligned}
\frac{R_{f}}{R_{\text {ref }}} & =\exp \left(\left(a_{2}-a_{1}\right)\left(\frac{1}{T}-\frac{1}{T_{\text {ref }}}\right)\right. \\
& \left.+\left(b_{2}-b_{1}\right)\left(\frac{1}{T^{2}}-\frac{1}{T_{\text {ref }}^{2}}\right)\right) .
\end{aligned}
$$

\section{Spectrosopic properties of pyrromethene 597-8C9}

\subsection{Influence of the solvent}

Pyrromethene 597-8C9 $\left(\mathrm{C}_{30} \mathrm{H}_{49} \mathrm{~N}_{2} \mathrm{BF}_{2}\right)$ manufactured by Exciton was selected because of its high solubility in alkanes, ketones or alcohols and the noticeable dependence on the temperature of the spectral shape of the fluorescence emission [10]. The fluorescence properties of this dye have been characterized in different liquids: 3-pentanone, ethanol and dodecane. Figure 1 shows the absorption spectra of

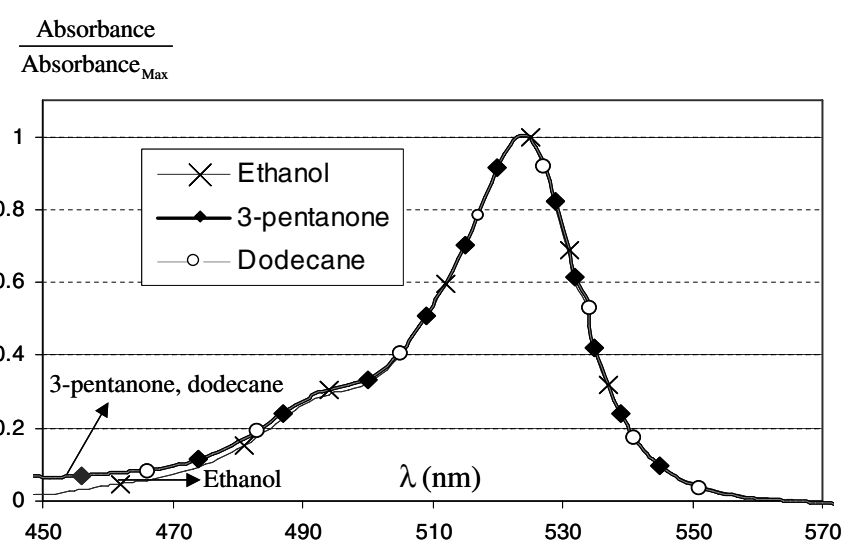

Figure 1. Absorption spectra of pyrromethene 597-8C9 dissolved in dodecane, ethanol and 3-pentanone $\left(T=25^{\circ} \mathrm{C}\right)$.

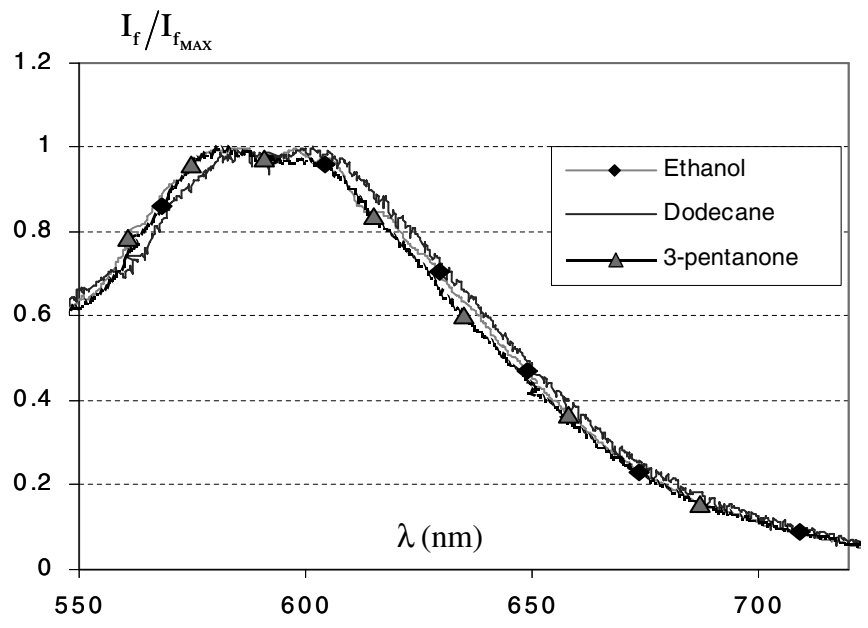

Figure 2. Fluorescence emission spectra of pyrromethene 597-8C9 dissolved in dodecane, ethanol and 3-pentanone $\left(T=25^{\circ} \mathrm{C}\right)$.

pyrromethene $597-8 \mathrm{C} 9$ at $25{ }^{\circ} \mathrm{C}$. The absorption spectrum of pyrromethene 597-8C9 dissolved in the three liquids is broadband and almost overlapping with a peak position almost independent of the solvent (about $524 \mathrm{~nm}$ for ethanol and 3-pentanone and $528 \mathrm{~nm}$ for dodecane). Consequently, the fluorescence can be easily induced by the green line of an argon ion laser at $514.5 \mathrm{~nm}$ or by a frequency-doubled Nd-Yag laser line $(\lambda=532 \mathrm{~nm})$. The fluorescence emission induced by a $514.5 \mathrm{~nm}$ laser line spreads over a large spectral band (figure 2). It exhibits an emission peak around $600 \mathrm{~nm}$ with a very moderate dependence on the solvent.

Additionally, mixtures made of 3-pentanone and ethanol have been considered. Generally, 3-pentanone is used with alkanes (e.g. iso-octane) or other fuels, since this ketone is well adapted to mimic the evaporation of real fuels particularly in automotive engines. The fluorescing properties of 3-pentanone in the gas phase can be used to determine the fuel vapour distribution [12]. Figure 3 presents the fluorescence spectra of pyrromethene 597-8C9 dissolved in different mixtures of ethanol and 3-pentanone. The spectra corresponding to pure ethanol and pure 3pentanone are superimposed in the same plot. The influence 


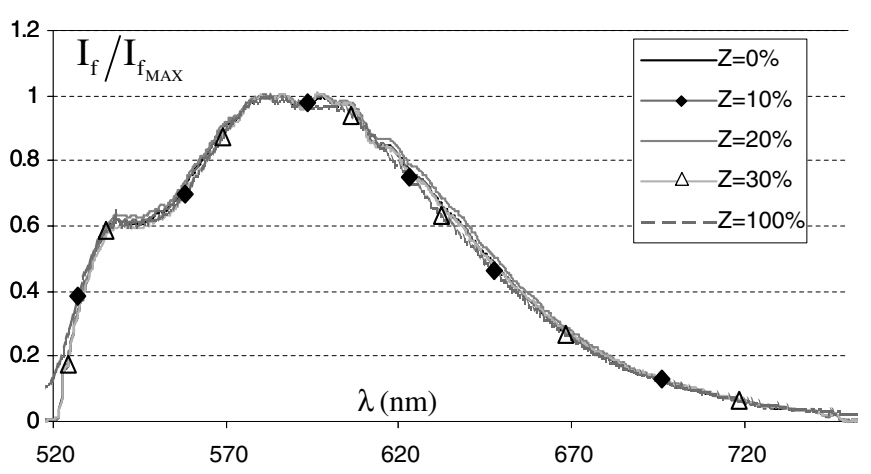

Figure 3. Influence of the composition on fluorescence spectra of mixtures made of 3-pentanone and ethanol $\left(T=25^{\circ} \mathrm{C}\right)$.

of the composition on the fluorescence spectra appears almost negligible.

\subsection{Influence of temperature on the fluorescence signal}

The temperature dependence of the fluorescence emission of an organic dye comes from two physical processes:

(1) the temperature dependence of the absorption crosssection at the laser wavelength, which is related to the Boltzmann distribution of the energy levels;

(2) the dependence of the quenching rate on temperature, following the Arrhenius law.

To estimate the relative importance of each of these phenomena, the influence of the temperature on the absorption can be easily quantified. The molar extinction coefficient $\varepsilon(\lambda)$ has been derived from absorption spectra measured by means of a CARY 500 SCAN spectrophotometer with a resolution of $1 \mathrm{~nm}$. The molar extinction coefficient is calculated according to Beer's law:

$$
\frac{I}{I_{0}}=\mathrm{e}^{-\varepsilon(\lambda) C l}
$$

where $I_{0}$ is the incident laser intensity, $I$ is the intensity after crossing the optical path $l$ and $C$ is the dye concentration. The dye volumetric concentration $C$ can be corrected from the thermal expansion of the medium in agreement with

$$
C(T)=\frac{C\left(T_{0}\right)}{1+\alpha T}
$$

where $\alpha$ is the temperature expansion coefficient of the liquid and $T_{0}$ is a reference temperature at which the concentration $C$ is known. Practically, $T_{0}$ is the ambient temperature at which the solutions are prepared.

The extinction coefficient is depicted as a function of the wavelength for the three solvents and for different temperatures in figure 4 . The range of tested temperatures is larger for dodecane, since its boiling point $\left(216^{\circ} \mathrm{C}\right)$ is higher than the other tested fuels, i.e. $78^{\circ} \mathrm{C}$ for ethanol and $102{ }^{\circ} \mathrm{C}$ for 3-pentanone. The absorption cross-section decreases moderately with the temperature for all three investigated solvents. This trend is more observable in the region of the peak of absorption (figure 5). The decay of the peak of absorbance is more important in the case of dodecane (about
$0.17 \%{ }^{\circ} \mathrm{C}^{-1}$ in the vicinity of $40{ }^{\circ} \mathrm{C}$ and about $0.1 \%{ }^{\circ} \mathrm{C}^{-1}$ for temperature higher than $100{ }^{\circ} \mathrm{C}$ ), whereas it follows very similar evolutions in the case of ethanol and 3-pentanone (about $0.15 \%{ }^{\circ} \mathrm{C}^{-1}$ ). From the above statements, it can be concluded that in the three investigated cases, the dependence on the temperature of the absorption cross-section at the laser wavelength is low. Furthermore, this study allows quantifying the fluorescence signal trapping due to re-absorption. The fluorescence emission spectrum at $25{ }^{\circ} \mathrm{C}$ is superimposed as shown in figure 4 to highlight the cross-talk between the absorption and emission spectra. As a result of this crosstalk, the fluorescence signal can be re-absorbed and this reabsorption is different for the two spectral bands of detection. The influence of this phenomenon on the fluorescence ratio needs to be carefully analysed (section 4).

In a further study, the emphasis was placed on the temperature sensitivity of the fluorescence emission spectral shape, characterized by $\beta(\lambda)$ in equation (1). This spectral shape is related to redistribution of population in the excited electronic state and to differences in quenching cross-sections and transition probabilities. The fluorescence is induced by the green line of the $\operatorname{argon}$ ion laser $(\lambda=514.5 \mathrm{~nm})$ and its spectrum has been recorded in a temperature-controlled glass wall cell. The cell, which is insulated by ceramic walls to avoid thermal losses, can be heated by means of a thermoelectric Peltier device (figure 6). Slits in the ceramic walls allow optical access for the laser beam and the fluorescence detection. To homogenize the temperature in the cell and to avoid photobleaching of the dye, the liquid is constantly agitated by means of a magnetic stirrer. A K-type thermocouple placed in the cell allows the liquid temperature to be measured with an accuracy of $\pm 0.1{ }^{\circ} \mathrm{C}$. The factor $\beta(\lambda)$ is determined according to equation (1), by calculating the ratio of two fluorescence spectra $I_{f 1}(\lambda)$ and $I_{f 2}(\lambda)$ recorded under the same optical conditions for two different temperatures $T_{1}$ and $T_{2}$ according to

$$
\beta(\lambda)=\ln \left(\frac{I_{f 1}(\lambda)}{I_{f 2}(\lambda)}\right) /\left(\frac{1}{T_{1}}-\frac{1}{T_{2}}\right) .
$$

Despite careful control of the measurement condition, slight changes in the optical alignment lead to a small translation of the value of $\beta(\lambda)$ from one experiment to another due to the fact that the ratio of the optical constants does not simply vanish as in equation (7). For this reason, it has been decided to impose an identical value to $\beta(\lambda)$ at an arbitrarily selected wavelength, presently $\lambda=560 \mathrm{~nm}$. The evolution of $\beta(\lambda)$ is presented in figure 7 for pyrromethene C97-8C9 dissolved in 3-pentanone, dodecane and ethanol. The trend is similar for the three solvents. The temperature sensitivity is low in a region ranging from $530 \mathrm{~nm}$ to $580 \mathrm{~nm}$, and then it increases sharply and reaches a plateau after $580 \mathrm{~nm}$. Furthermore, the spectral sensitivity of ethanol and 3-pentanone almost overlap whereas dodecane exhibits a slightly larger change in temperature sensitivity.

To give a more comprehensive description, mixtures of 3 -pentanone and ethanol have also been considered. The temperature sensitivity factor $\beta(\lambda)$ is depicted in figure 8 for different volume fractions of 3-pentanone. It can be observed that the composition has little influence on the evolution of $\beta(\lambda)$. This is a particularly interesting feature when dealing 

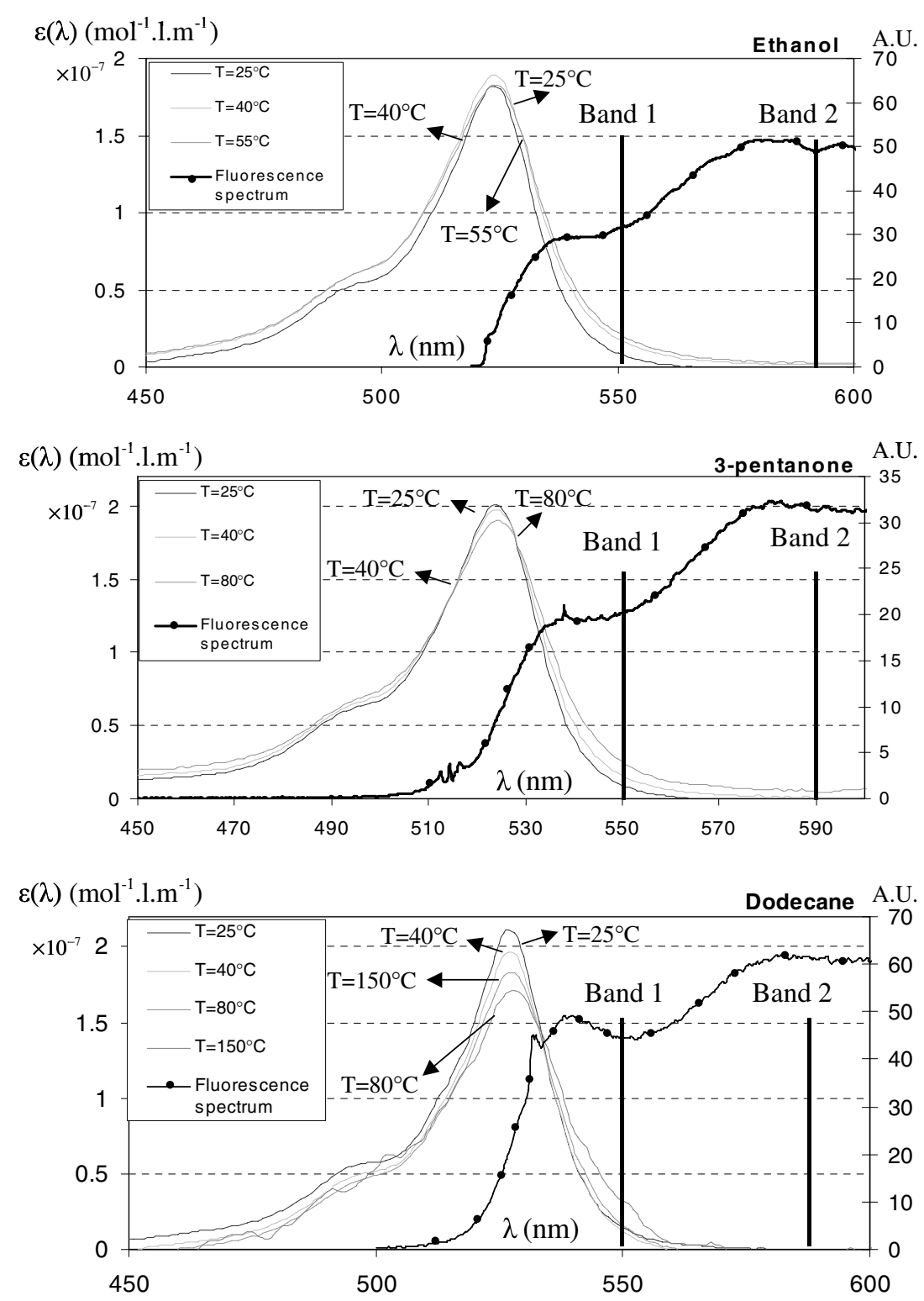

Figure 4. Influence of the temperature on the absorption spectrum (molar extinction coefficient) of pyrromethene 597-8C9 dissolved in 3-pentanone, dodecane and ethanol. Fluorescence spectra are superimposed to visualize the crosstalk region.

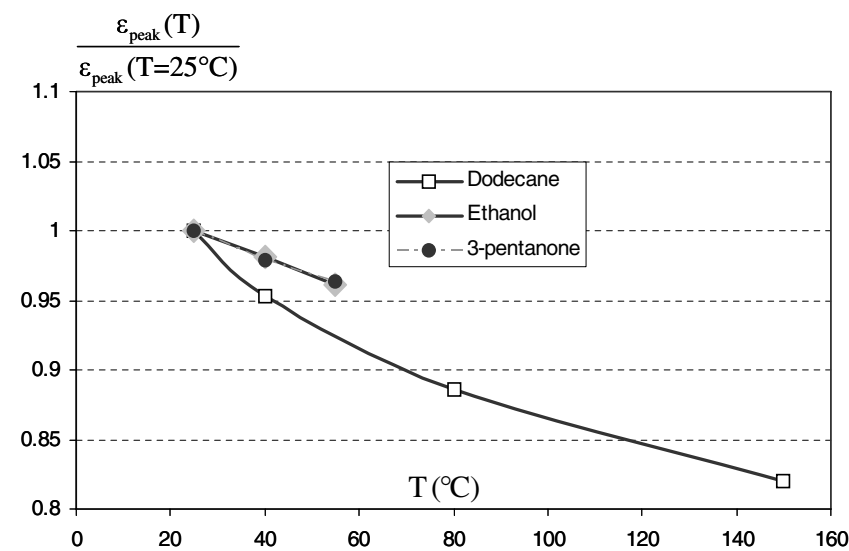

Figure 5. Evolution of the molar absorption coefficient at the absorption peak as a function of temperature, for the different investigated solvents (results are normalized by the value at $\left.T=25^{\circ} \mathrm{C}\right)$.

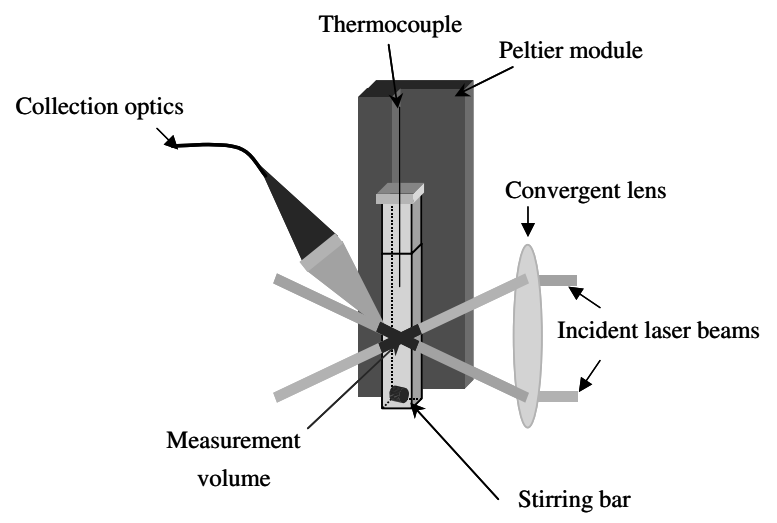

Figure 6. Sketch of the temperature-controlled calibration cell.

with evaporating mixtures that undergo a change of composition. Since the composition of the liquid does 


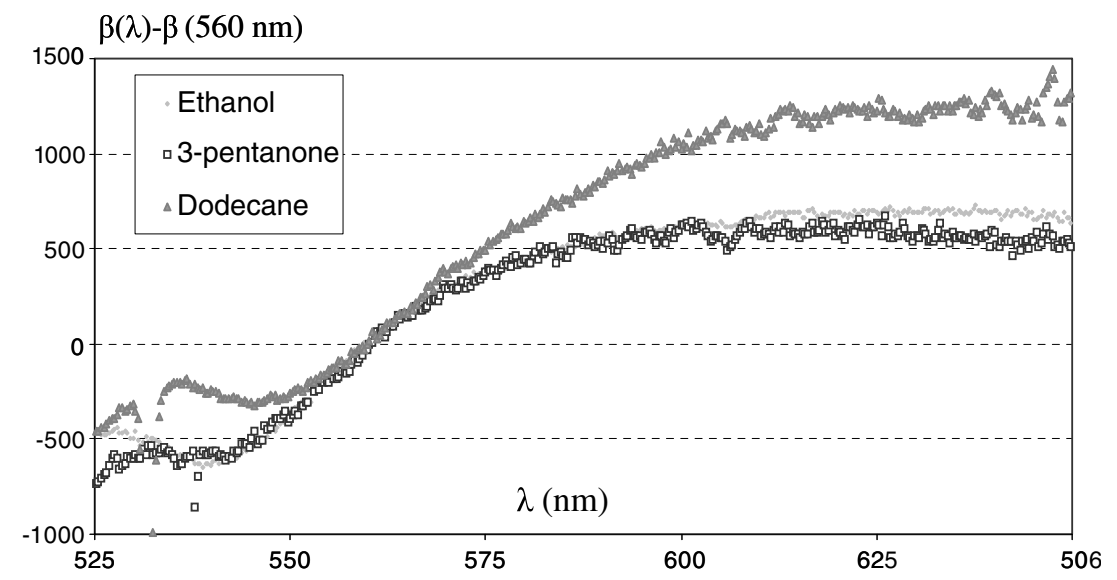

Figure 7. Evolution of the temperature sensitivity $\beta(\lambda)$ as a function of the wavelength for pyrromethene 597-8C9 dissolved in 3-pentanone, dodecane and ethanol.

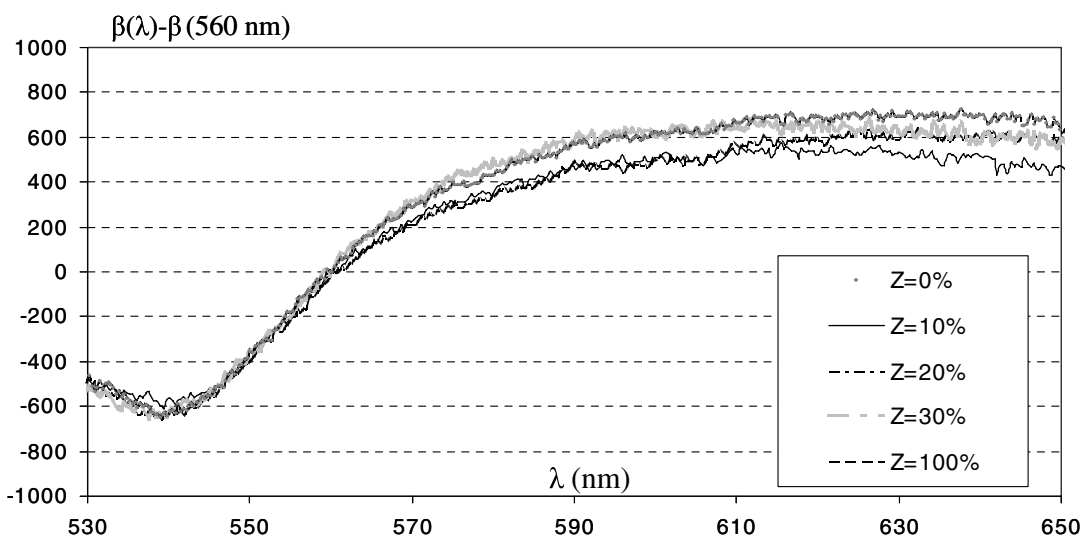

Figure 8. Evolution of the temperature sensitivity of pyrromethene 597-8C9 as a function of the wavelength for different mixtures of 3-pentanone and ethanol.

not affect the temperature sensitivity of the dye and the fluorescence signal level, measurements can be performed with the same ease as in the case of a single component fuel. In contrast, the fact that rhodamine B does not share this behaviour was a serious drawback in studying the evaporation of bicomponent droplets made of ethanol and acetone [9].

\section{Experimental set-up}

\subsection{Optical components and data processing}

To achieve accurate measurements of the temperature, two spectral bands of detection should be selected in such a way that their temperature sensitivity is as different as possible. The availability of the interference filters on the market has fixed the choice on

- band 1: (540 nm; $560 \mathrm{~nm})$ (spectral width: $20 \mathrm{~nm})$

- band 2: (590 nm; $610 \mathrm{~nm})$ (spectral width: $20 \mathrm{~nm}$ ).

One important feature of this selection is that the fluorescence intensity of the two bands is relatively high and well balanced. The situation would be quite different if rhodamine B was used: the signal level would be very low for the band located in the green-yellow part of the spectrum [6].

It is also possible to estimate the influence on the fluorescence ratio of the re-absorption of the fluorescence signal due to the partial overlap of the absorption and fluorescence spectra. This calculation is carried out using Beer's law (equation (5)), with $\varepsilon\left(\lambda_{c}\right)$ coming from the preliminary spectroscopic study (section 3.2). In the present case, $\lambda_{c}$ is the central wavelength of the two spectral bands, $550 \mathrm{~nm}$ and $600 \mathrm{~nm}$, respectively. The values of the extinction coefficient are $10^{-6} \mathrm{~mol}^{-1} 1 \mathrm{~m}^{-1}$ and $5 \times 10^{-5} \mathrm{~mol}^{-1} 1 \mathrm{~m}^{-1}$ at $550 \mathrm{~nm}$ and $600 \mathrm{~nm}$, respectively. By considering an optical path of about $100 \mu \mathrm{m}$ which approximately corresponds to the droplet diameter in the experiments, knowing also that the dye concentration is equal to $10^{-6} \mathrm{~mol}^{-1}$, the influence of the fluorescence re-absorption on the fluorescence ratio remains less than $0.004 \%$ which can be neglected.

The fluorescence is induced by an argon ion laser tuned on its green line $\lambda=514.5 \mathrm{~nm}$. A LDV beam system makes possible simultaneous measurements of the temperature and velocity of the droplets which is the scope of further experimental developments. The fluorescence signal is focused on an optical fibre by an achromatic doublet. Then, a set of dichroic and optical filters enables us to divide the 


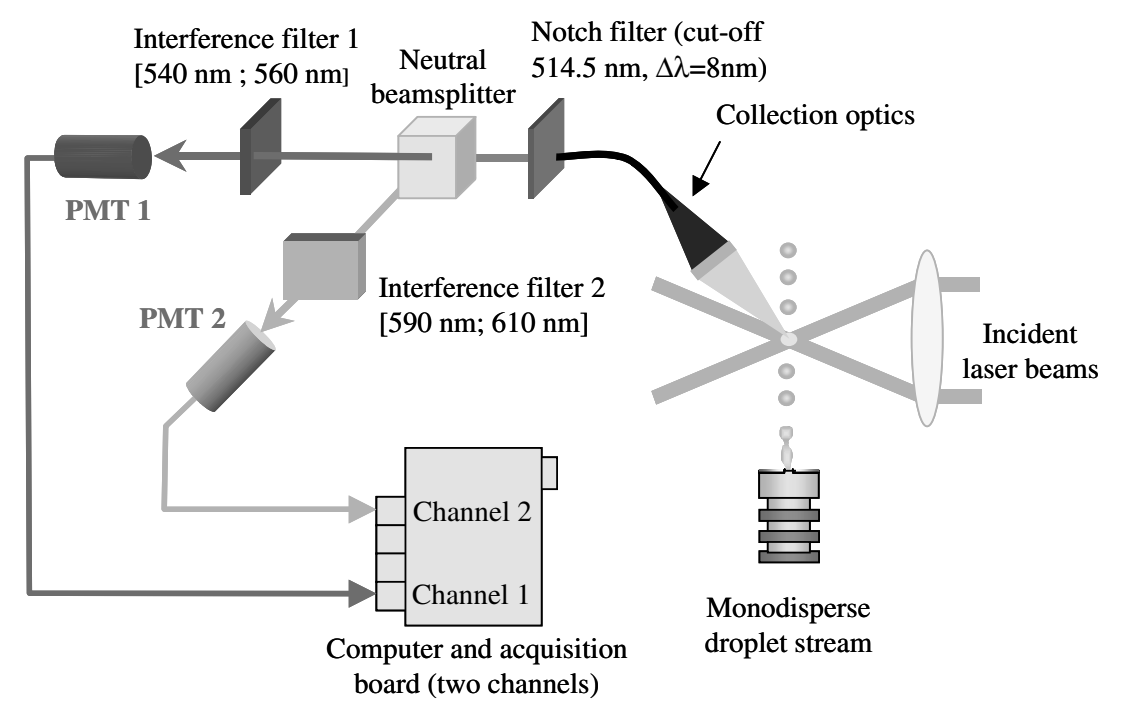

Figure 9. Sketch of the optical device.

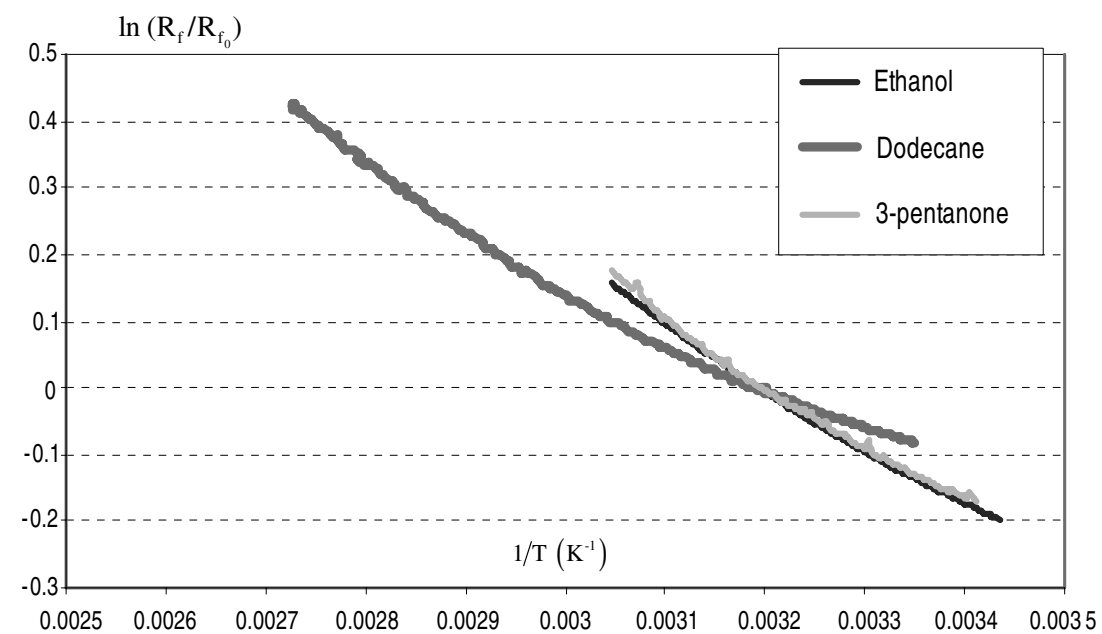

Figure 10. Evolution of the fluorescence ratio $R_{f}$ as a function of temperature for pyrromethene dissolved in ethanol, 3-pentanone and dodecane. The fluorescence ratio is normalized by its value at $T=40^{\circ} \mathrm{C}$.

fluorescence signal into the two specified spectral bands of detection (figure 9). The scattered laser light is eliminated with the use of a notch filter (Super Notch Plus, Kayser Optical, optical density 6). Fluorescence is detected in the two spectral bands by means of two photomultipliers equipped with fastresponding pre-amplifiers followed by analogue filters (cutoff frequency: $30 \mathrm{kHz}$ ). The experimental set-up is relatively compact and can be contained in a box which is $40 \mathrm{~cm}$ long and $30 \mathrm{~cm}$ wide. The measurement volume, resulting from the intersection of the laser beams and the image of the core of the optical fibre, is about $200 \mu \mathrm{m}$ in the laser beams' direction and $150 \mu \mathrm{m}$ in the transverse direction.

\subsection{Calibration of the fluorescence ratio as a function of temperature}

Calibrations are required to capture the response of the detection system to a variation of the temperature. They are performed in the temperature-controlled cell described in section 3.2. The temperature in the cell is progressively increased and the fluorescence signal is recorded in the two spectral bands of interest. The temperature increase is sufficiently low to average both fluorescence intensities and thermocouple voltage over $2 \mathrm{~s}$. Then the uncertainty in the thermocouple measurement was estimated at about $0.1{ }^{\circ} \mathrm{C}$ in the considered temperature range. Finally, the fluorescence ratio $R_{f}$ is calculated and the evolution of $\ln \left(R_{f}\right)$ is displayed as a function of $1 / T$.

The signal acquisition at $2 \mathrm{MHz}$ enables us to achieve a rather good statistical convergence in the averaging process of the fluorescence intensity. The ratio between the RMS deviation of the measurements and their mean is less than $0.12 \%$, which leads to a possible error of $0.25 \%$ in the fluorescence ratio.

Figure 10 shows the results in the case of the pure components. For reasons of clarity, the results have been 


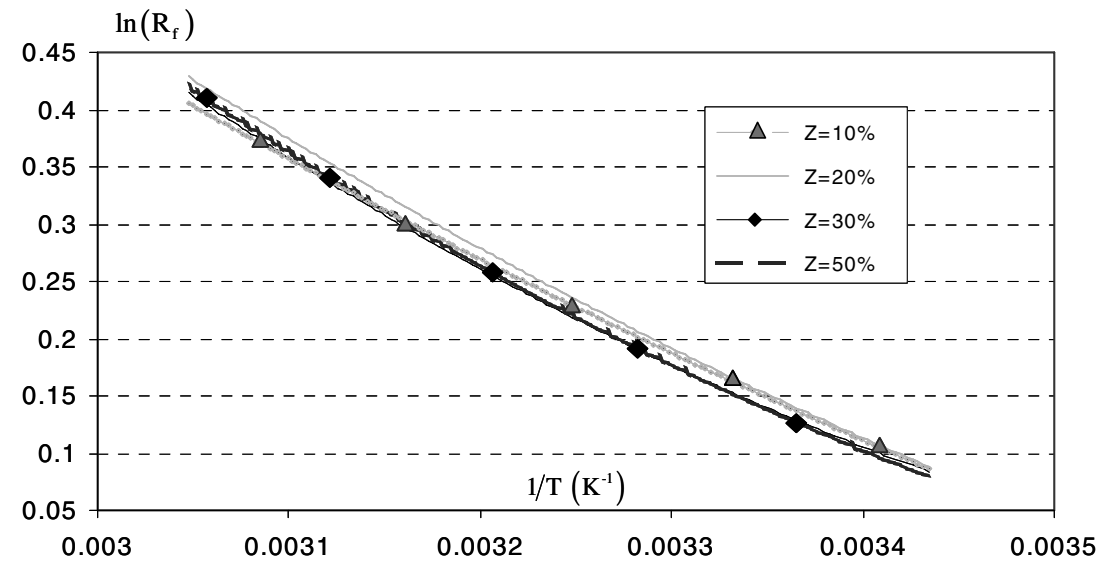

Figure 11. Evolution of the fluorescence ratio $R_{f}$ as a function of temperature for pyrromethene dissolved in the ethanol/3-pentanone mixture ( $Z$ is the volume fraction of 3-pentanone).

Table 2. Calibration coefficients $\left(a_{1}-a_{2}\right),\left(b_{1}-b_{2}\right)$ and their respective uncertainties.

\begin{tabular}{lllll}
\hline Solvent & $A=a_{1}-a_{2}$ & $\Delta A / A(\%)$ & $B=b_{1}-b_{2}$ & $\Delta B / B(\%)$ \\
\hline Ethanol or 3-pentanone & 609027 & 2.2 & -5119 & 1.6 \\
Dodecane & 682202 & 1.8 & -4939 & 1.2 \\
\hline
\end{tabular}

normalized by the fluorescence ratio measured at a designated temperature $\left(T=40^{\circ} \mathrm{C}\right)$. It appears that the rate of variation of the fluorescence ratio is about $1.3 \% \mathrm{~K}^{-1}$ for 3-pentanone and ethanol, as this sensitivity depends more evidently on the temperature level for dodecane: it is about $0.4 \% \mathrm{~K}^{-1}$ at $25{ }^{\circ} \mathrm{C}$ and it can reach $1.51 \% \mathrm{~K}^{-1}$ for temperatures on the order of $100{ }^{\circ} \mathrm{C}$. Taking into consideration the results of section 3.2 concerning the temperature dependence of the absorption cross-section, it can be concluded that the temperature sensitivity of the pyrromethene 597-8C9 can be mainly attributed to the temperature dependence of the quenching rate. Results related to the mixture of ethanol/3pentanone are displayed in figure 11 . For all the tested volume fractions of 3-pentanone, the calibration curves are almost superimposed. The effect of the moderate deviation between these different curves on the temperature inversion process can be assessed. In the worst case, the deviation leads to a variation of about $\pm 1.1{ }^{\circ} \mathrm{C}$ in the range $\left[20-70{ }^{\circ} \mathrm{C}\right]$ for extreme composition ranging from $Z=10 \%$ to $50 \%$. As a result, a unique pair of coefficients $\left(a_{1}-a_{2}\right)$ and $\left(b_{1}-b_{2}\right)$ can be used for these mixtures in the data processing for most of the applications.

However, this feature is not general. For instance, one can anticipate that calibration curves would not be superimposed in the case of mixtures of dodecane with ethanol or 3pentanone since the temperature sensitivity of the fluorescence of pyrromethene 597-8C9 is different when dissolved in dodecane compared to ethanol or 3-pentanone (figure 7).

A way to estimate the error in the calibration coefficients $\left(a_{1}-a_{2}\right)$ and $\left(b_{1}-b_{2}\right)$ consists in finding, among the secondorder polynomials entering the confidence interval of the fluorescence ratio, those two that are the more distant, and comparing their coefficients. The values of $\left(a_{1}-a_{2}\right),\left(b_{1}-\right.$ $b_{2}$ ) and their corresponding uncertainties are given in table 2 .

\section{Experimental validation: application to temperature measurements of droplets}

\subsection{Experimental set-up}

Linear monodisperse droplet streams are generated by Rayleigh disintegration of a liquid jet undergoing vibrations generated by a piezoelectric ceramic. The voltage applied to the piezoceramic is a square wave, the amplitude of which determines the position of the break-up zone for a given fuel at a given temperature. The fuel is pre-heated in the injector body by means of a heat exchanger supplied by circulating water. The temperature of the fuel is measured exactly at the injection point by means of a K-type thermocouple. For specific frequencies of the piezoceramic, the liquid jet breaks up into equally spaced and monosized droplets [13]. By adjusting the liquid flow rate and the piezoceramic frequency, it is possible to increase the droplet spacing up to about six times the droplet diameter. In order to investigate the droplet heating and evaporation for different fuels or fuel mixtures having different volatilities, the droplets are then injected into an enclosure fed with hot air issuing from an electrical heater (figure 12). The air flowrate can be adjusted and its value is measured upstream from the heater. In order to limit the thermal losses and to homogenize the temperature in the enclosure, a resistive electrical wire is inserted within the enclosure wall so that the wall temperature can be regulated to match that of the entering air. Temperature up to $370^{\circ} \mathrm{C}$ can be reached with this system. Additionally, glass windows have been mounted on the walls for optical access. Destabilization of the droplet stream by the air motion may be a critical issue in this experiment. The air velocity is therefore maintained between $0.1 \mathrm{~m} \mathrm{~s}^{-1}$ and $0.2 \mathrm{~m} \mathrm{~s}^{-1}$ and the air flow is quietened by forcing it through metallic foam sandwiched between two drilled walls. The problem of vapour saturation must be 


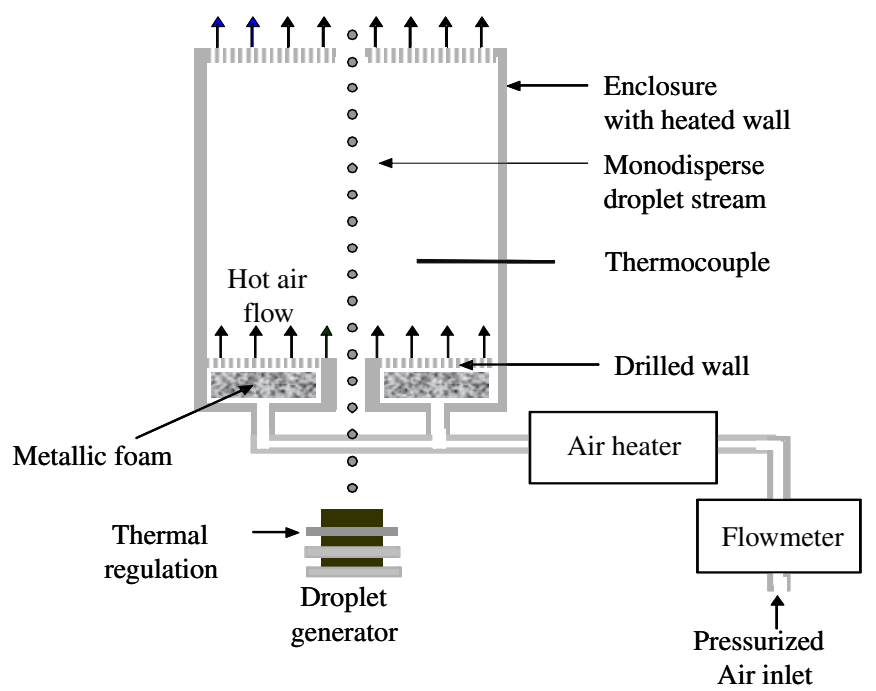

Figure 12. Layout of the heated enclosure and the droplet generator.

considered carefully due to the moderate air blowing and the finite dimension of the chamber which has an inner diameter of $10 \mathrm{~cm}$ and a height of $14 \mathrm{~cm}$. An estimate of the diffusion length $L$ can be obtained by considering a diffusivity $\alpha=10^{-5}$ $\mathrm{m}^{2} \mathrm{~s}^{-1}$ for the vapour and a diffusion duration $t$ equal to $1 \mathrm{~s}$, corresponding roughly to the residence time of the vapour in the cell. The diffusion length, which is on the order of $\sqrt{\alpha t}$, is about $3 \mathrm{~mm}$. This length is negligible compared to the inner radius of the enclosure, which ensures non-saturated conditions.

\subsection{Uncertainty study}

An estimate of the measurement accuracy is provided here. The different sources of measurement errors can be identified as follows:

- the limited number of samples in the averaging process when calculating the fluorescence ratio: $\Delta R / R=0.25 \%$,

- the reference determination: the thermocouple accuracy $\Delta T_{\text {ref }}=0.1^{\circ} \mathrm{C}$ and the error $\Delta R_{\text {ref }}$ on the reference ratio is equal to $\Delta R$ and

- the accuracy of the calibration coefficients (see table 2).

According to equation (4), the temperature uncertainty can be given in the following form:

$$
\begin{aligned}
\Delta T & =\left|\frac{\partial T}{\partial R}\right| \Delta R+\left|\frac{\partial T}{\partial R_{\mathrm{ref}}}\right| \Delta R_{\mathrm{ref}}+\left|\frac{\partial T}{\partial T_{\mathrm{ref}}}\right| \Delta T_{\mathrm{ref}} \\
& +\left|\frac{\partial T}{\partial A}\right| \Delta A+\left|\frac{\partial T}{\partial B}\right| \Delta B
\end{aligned}
$$

where $A=a_{2}-a_{1}$ and $B=b_{2}-b_{1}$ correspond to the pair of coefficients in equation (4).

Among all these sources of error, $\Delta R$ is the only one that affects the reproducibility of the measurements. Other sources result in a systematic error. Typically, the error in the temperature can be estimated from equation (8) to about $1.5^{\circ} \mathrm{C}$ with only $0.3{ }^{\circ} \mathrm{C}$ corresponding to problems of reproducibility.
Table 3. Injection conditions for the different tested pure components.

\begin{tabular}{lccc}
\hline Fuel & Dodecane & 3-Pentanone & Ethanol \\
\hline$T_{\text {air }}\left({ }^{\circ} \mathrm{C}\right)$ & 370 & 370 & 370 \\
$D_{0}(\mu \mathrm{m})$ & 107 & 118 & 118 \\
$C_{0}$ & 4.6 & 4.8 & 4.7 \\
$V_{0}\left(\mathrm{~m} \mathrm{~s}^{-1}\right)$ & 10 & 11.5 & 11 \\
$T_{0}\left({ }^{\circ} \mathrm{C}\right)$ & 25.1 & 25.3 & 25 \\
\hline
\end{tabular}

\subsection{Experimental results}

A reference measurement is required to obtain quantitative measurements of the temperature as specified by equation (4). Practically, it can be performed at room temperature close to the outlet of the injector on the liquid jet that disintegrates into the droplets. Under these conditions, the temperature of the liquid can be safely assumed to be equal to that measured in the injector body by a thermocouple. When measuring the droplet temperature, the signal of the two spectral bands is averaged over the transit time of the droplet in the probe volume. The fluorescence ratio is then calculated and converted into temperature according to equation (4). About 10000 droplets are necessary to obtain a satisfactory convergence of the mean temperature.

During the experiments in the hot air chamber, the injection temperature is maintained around $25^{\circ} \mathrm{C}$. The droplet velocity is measured by using a laser Doppler anemometry system. Provided the measurement of the droplet velocity, the distance $z$ from the injector can be converted into time according to $t=\int_{0}^{z} \frac{\mathrm{d} u}{V(u)}$. The initial diameter $D_{0}$ is determined by the measurement of the fuel flowrate $Q$ at the injector exit:

$$
D_{0}=(6 Q / \pi f)^{1 / 3}
$$

where $f$ denotes the piezoceramic frequency. The injection conditions are almost identical for all the investigated cases, although moderate variations could not be avoided due to the difference of fuel viscosities and surface tensions that considerably modify the droplet disintegration process. The initial conditions are summarized in table $3 . C_{0}$ corresponds to the ratio between the droplet spacing and diameter.

Droplets made of pure components are investigated first in a quiescent atmosphere at ambient temperature. Figure 13 shows the droplet change in temperature as a function of time for 3-pentanone, ethanol and dodecane. The temperature decrease due to evaporation is moderate and corresponds to about $1{ }^{\circ} \mathrm{C}$ for dodecane, $1.5{ }^{\circ} \mathrm{C}$ for 3 -pentanone and $2{ }^{\circ} \mathrm{C}$ for ethanol in a period of about $10 \mathrm{~ms}$. As expected, droplets made of 3-pentanone and ethanol, which are much more volatile than dodecane, undergo a more important cooling.

The same experiment has been carried out in air heated at $T_{\text {air }}=370{ }^{\circ} \mathrm{C}$. However, the analysis of the results is a little more difficult in this case, since the initial conditions are less easy to control. Before entering the heated chamber, the droplets go through a hole drilled in the wall of the enclosure. The small size of this hole modifies the boundary conditions and the heat and mass transfers. To compare the results, the temperature is reduced by the temperature of the first measurement point inside the enclosure. In this way, 


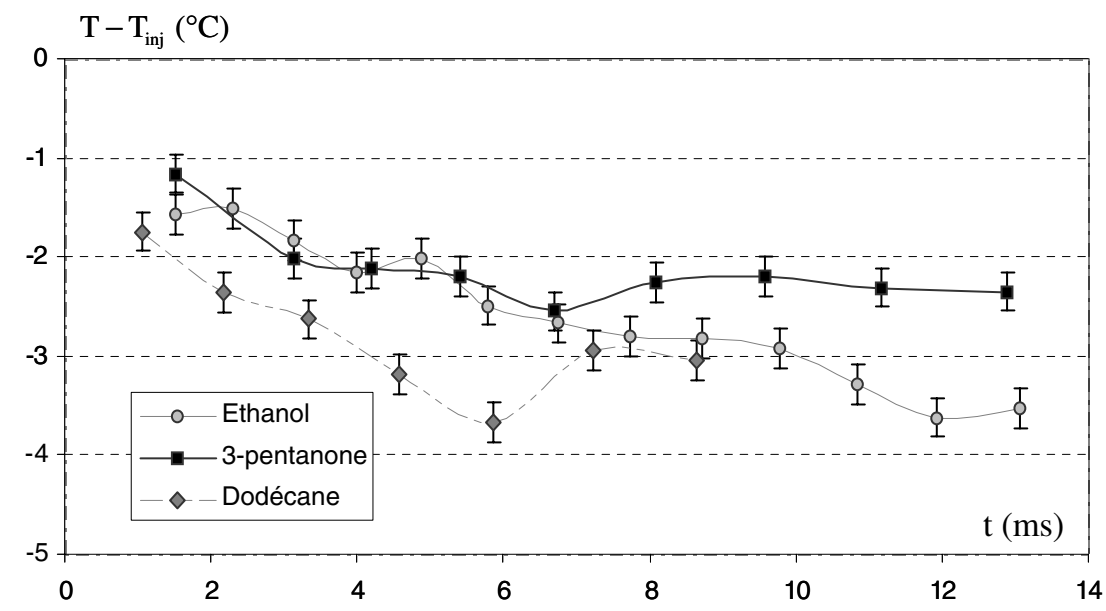

Figure 13. Temporal evolution of the droplet temperature (ethanol, 3-pentanone and dodecane) at ambient temperature ( $T_{\text {inj }}$ is the fuel injection temperature).

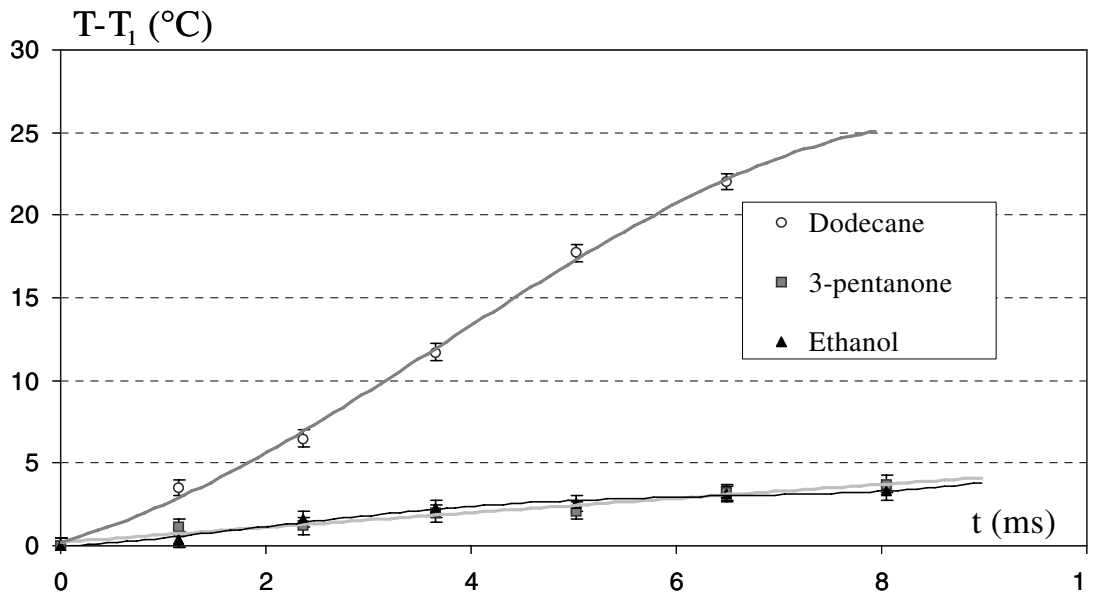

Figure 14. Temporal evolution of the droplet temperature (ethanol, 3-pentanone and dodecane) with $T_{\text {air }}=370{ }^{\circ} \mathrm{C}\left(T_{1}\right.$ is the droplet temperature at the first measurement point).

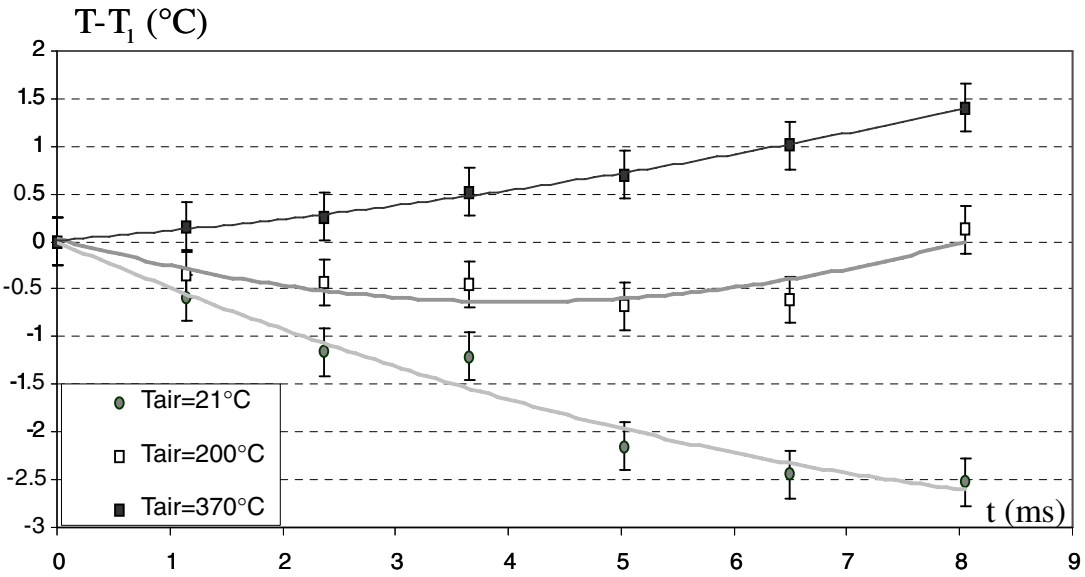

Figure 15. Temporal evolution of the droplet temperature for 3-pentanone/ethanol $(Z=50 \%)$ for different air temperatures $\left(T_{1}\right.$ is the droplet temperature at the first measurement point).

the droplet heating is characterized (figure 14) and it can be verified that the heating rate increases with decreasing product volatility (about $0.41 \mathrm{~K} \mathrm{~ms}^{-1}$ for ethanol, $0.47 \mathrm{~K} \mathrm{~ms}^{-1}$ for 3-pentanone and $3 \mathrm{~K} \mathrm{~ms}^{-1}$ for dodecane). 


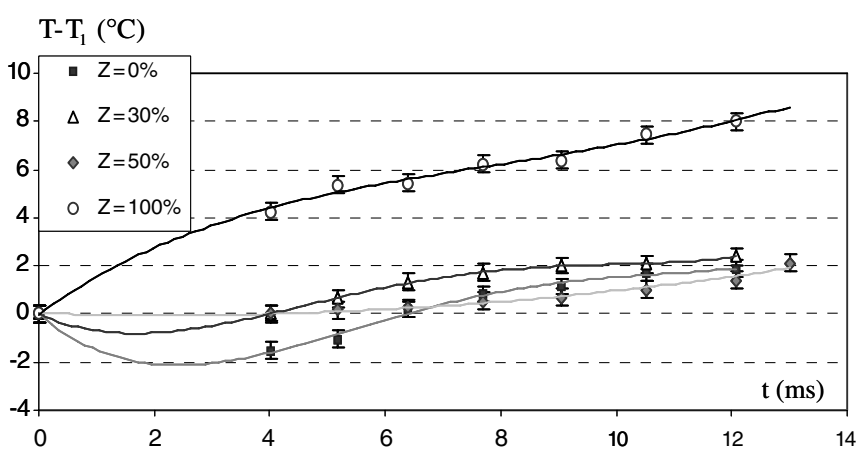

Figure 16. Temporal evolution of the droplet temperature for different compositions of the 3-pentanone/ethanol mixture, with $T_{\text {air }}=370^{\circ} \mathrm{C}\left(T_{1}\right.$ is the droplet temperature at the first measurement point).

The effect of the air temperature was studied on a mixture containing $50 \%( \pm 0.3 \%)$ of 3-pentanone and $50 \%( \pm 0.3 \%)$ of ethanol. The air temperature was increased from $21{ }^{\circ} \mathrm{C}$ to $370{ }^{\circ} \mathrm{C}$ (figure 15). When the fuel is injected at ambient temperature $\left(T_{\text {air }}=21{ }^{\circ} \mathrm{C}\right)$, a cooling is observed due to the domination of the latent heat over the other thermal fluxes. This cooling is still observed at $T_{\text {air }}=200{ }^{\circ} \mathrm{C}$, but it is limited to the first $5 \mathrm{~ms}$ of the observation period. Afterwards, the convective heat flux becomes large enough to balance the thermal losses due to evaporation and heats up the droplet. When $T_{\text {air }}$ is equal to $370{ }^{\circ} \mathrm{C}$, only a heating phase can be observed.

Finally, measurements of the droplet temperature were performed on the ethanol/3-pentanone mixture. Figure 16 depicts the evolution of the droplet temperature for mixtures containing $Z=0 \%, 30 \%, 50 \%( \pm 0.3 \%)$ and $100 \%$ of 3 pentanone at an ambient temperature of $370{ }^{\circ} \mathrm{C}$.

As expected, droplets made of pure 3-pentanone experience the more important increase in their temperature. After a moderate cooling of about $2{ }^{\circ} \mathrm{C}$, droplets made of pure ethanol are heated up at almost the same rate as 3pentanone droplets. The curve related to $Z=30 \%( \pm 0.3 \%)$ lies between those of $Z=0 \%$ and $100 \%$, but the heating seems to proceed at a lower rate in the case of $Z=50 \%( \pm 0.3 \%)$. An explanation for that lies in the possibly non-ideal behaviour of the 3-pentanone/ethanol mixture, which is not unusual when mixings between ketones and alcohols are concerned [14].

\section{Conclusions}

Pyrromethene 597-8C9 has been used to measure the temperature of droplets with the two-colour laser-induced fluorescence technique. It has been demonstrated that pyrromethene 597-8C9 is a flexible tracer in the sense that it can be introduced into several fuel types such as alkanes, ketones or alcohols without changing the spectral bands of detection and the optical layout. Furthermore, mixtures made of 3-pentanone and ethanol have been investigated: the fluorescence signal was found to be independent of the composition of the fuel. This behaviour leads the way to further investigations of multicomponent fuels such as primary reference fuels (PRF) that undergo composition and temperature changes simultaneously. The fluorescence of the dyes dissolved in liquids is generally not sensitive to the pressure in the pressure range usually encountered in engines (less than $5 \mathrm{MPa}$ ). Pressure-induced shifts of the fluorescence spectra of dyes can be observed around largely higher pressure levels, typically around a few GPa $[15,16]$.

Consequently, this tracer should be suitable for spray combustion studies under engine conditions, i.e. in a flame, and under a pressure which can reach $5 \mathrm{MPa}$. The main limitation is the thermal dissociation of the tracer; to the best of our knowledge, the tracer has been tested up to the boiling point of dodecane (about $216^{\circ} \mathrm{C}$ ), without any damage. However, the boiling temperature of pyrromethene 597-8C9 is about $250{ }^{\circ} \mathrm{C}$, which may limit the use of the dye for products even less volatile than dodecane.

\section{References}

[1] Coolen M C J, Kieft R N, Rindt C C and van Steenhoven A A 1999 Application of 2-D LIF temperature measurements in water using a Nd: YAG laser Exp. Fluids 27 420-6

[2] Lemoine F, Antoine Y, Wolff M and Lebouché M 1999 Simultaneous temperature and 2D velocity measurements in a turbulent heated jet using combined laser-induced fluorescence and LDA Exp. Fluids 28 315-23

[3] Copetta J and Rogers C 1998 Dual emission laser induced fluorescence for direct planar scalar behavior measurements Exp. Fluids 25 1-15

[4] Sakakibara J and Adrian R 1999 Whole field measurements of temperature in water using two-color laser induced fluorescence Exp. Fluids 26 7-15

[5] Sakakibara J and Adrian R J 2004 Measurement of temperature field of Rayleigh-Bénard convection using two-color laser-induced fluorescence Exp. Fluids 37 331-40

[6] Lavieille P, Lemoine F, Lebouché M and Lavergne G 2001 Evaporating and combusting droplet temperature measurement using two colors laser-induced fluorescence Exp. Fluids 31 45-55

[7] Lavieille P, Lemoine F, Lebouché M and Lavergne G 2001 Mesure de la température de gouttelettes en combustion par fluorescence induite par laser à deux couleurs: résultats préliminaires et perspectives $C$. R. Acad. Sci., Paris II b 329 557-64

[8] Viriot M L and Andre J C 1989 Fluorescent dyes: a search for new tracers for hydrology Analusis 17 97-104

[9] Maqua C, Castanet G, Doué N, Lavergne G and Lemoine F 2006 Temperature measurements of binary droplets using three color laser-induced fluorescence Exp. Fluids 40 786-97

[10] Wolff M, Delconte A, Schmidt F, Gucher P and Lemoine F 2007 High-pressure Diesel spray temperature measurements using two-colour laser-induced fluorescence Meas. Sci. Technol. 18 697-706

[11] Castanet G, Lavieille P, Lebouché M and Lemoine F 2003 Measurement of the temperature distribution within monodisperse combusting droplets in linear stream using two colors laser-induced fluorescence Exp. Fluids 35 563-71

[12] Han D and Steeper R 2002 Examination of iso-octane/ketone mixtures for quantitative LIF measurements in a DSI engine SAE Technical Paper No 2002-02-0837

[13] Frohn A and Roth N 2000 Dynamics of Droplets (Berlin: Springer) 
[14] Campbell S W, Wilseak R A and Thodos G 1987 Vapor-liquid equilibrium measurements for the ethanol-acetone system at 372.7, 397.7 and 422.6 K J. Chem. Eng. Data 32 357-62

[15] Zang B, Chandrasekhar M and Chandrasekhar H R 1985 Pressure-induced shifts of the fluorescence spectrum of rhodamine $6 \mathrm{G}$ in solution Appl. Opt. 24 2779-86

[16] Costa T M H, Hoffmann H S, Benvenutti E V, Stefani V and Gallas M R 2005 Pressure-induced changes on the optical properties and microstructure of silica-gel matrices doped with rhodamine 6G Opt. Mater. 27 1819-24 